\title{
COMPRESSIVE SENSING BASED IMAGING VIA BELIEF PROPAGATION
}

\author{
By
}

Preethi Modur Ramachandra

Approved:

Mina Sartipi

Associate Professor of Computer Science

(Director of Thesis)

Raziq Yaqub

Associate Professor of Electrical Engineering (Committee Member)
Stephen D. Craven

Assistant Professor of Electrical Engineering (Chair Person)
William H. Sutton

Dean of the College of Engineering and Computer Science
A. Jerald Ainsworth

Dean of the Graduate School 


\title{
COMPRESSIVE SENSING BASED IMAGING VIA BELIEF PROPAGATION
}

By

Preethi Modur Ramachandra

\author{
A Thesis \\ Submitted to the Faculty of the \\ University of Tennessee at Chattanooga \\ in Partial fulfillments of the Requirements \\ for the degree of Master of Science \\ in Electrical Engineering
}

The University of Tennessee at Chattanooga Chattanooga, Tennessee

May 2012 


\begin{abstract}
Multiple description coding (MDC) using Compressive Sensing (CS) mainly aims at restoring an image from a small subset of samples with reasonable accuracy using an iterative message passing decoding algorithm commonly known as Belief Propagation (BP). The CS technique can accurately recover any compressible or sparse signal from a lesser number of nonadaptive, randomized linear projection samples than that specified by the Nyquist rate.

In this work, we demonstrate how CS-based encoding generates measurements from the sparse image signal and the measurement matrix. Then we demonstrate how a BP decoding algorithm reconstructs the image from the measurements generated. In our work, the CS-BP algorithm assumes that all the unknown variables have the same prior distribution as we do not have any knowledge of the side information available during the initiation of the decoding process. Thus, we prove that this algorithm is effective even in the absence of side information.
\end{abstract}




\section{DEDICATION}

I dedicate this work to my parents, Ramachandra and Sharadamba, for their love, encouragement, support and for being a great source of inspiration and motivation all through my life. 


\section{ACKNOWLDEGEMENTS}

I offer my sincerest gratitude to my advisor Dr. Mina Sartipi. I honestly appreciate all her contributions of time, ideas and funding to make my dream of attending graduate school possible and moreover in making this experience productive and interesting. Her encouragement, thoughtful criticism and invaluable assistance from the initial to the final level enabled me in accomplishing this task successfully. You are a true inspiration and it has been an honor to work with you.

I would also like to acknowledge the other members of my thesis committee, namely, Dr. Stephen D. Craven and Dr. Raziq Yaqub for their invaluable aid and direction provided throughout the intricacies of the master's program and thesis process.

Special thanks are due to my family in India, especially my parents; I couldn't have accomplished all this without their encouragement and endless love. They have given me their unequivocal support throughout, as always, for which my mere expression of thanks likewise does not suffice. I owe my deepest gratitude to them and my cousin Harsha and his wife Shweta, for their moral support, guidance and love throughout.

My deepest thanks to my dearest and very special friend Swaroop for being with me in times of joy and sorrow and in making this journey a successful one.

Also, I am very grateful to my manager Anne Robinson who has been very understanding and motivating at all times, my colleagues, fellow graduate students, my close friends Sushma, 
Sharmila, Khushbu and Ramya for their friendship, words of encouragement and for being a constant source of support both morally and emotionally which always made me feel at home.

Lastly, I offer my regards to all of those who have been a part of this amazing journey and supported me in any respect during the completion of this thesis. 


\section{TABLE OF CONTENTS}

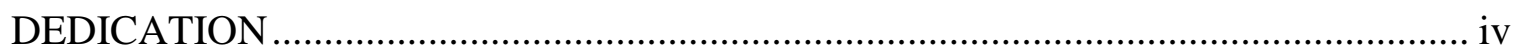

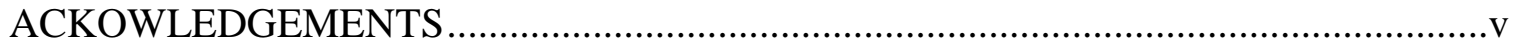

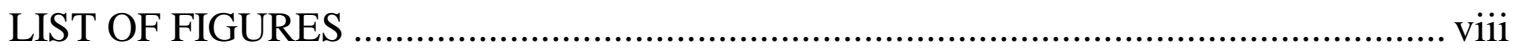

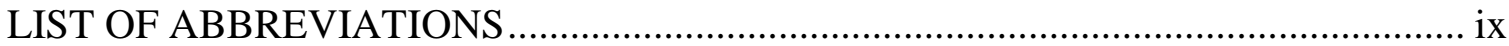

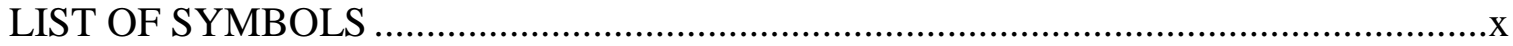

\section{CHAPTER}

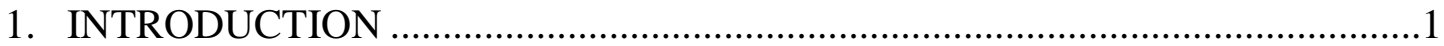

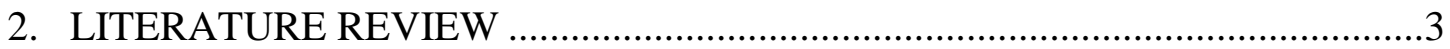

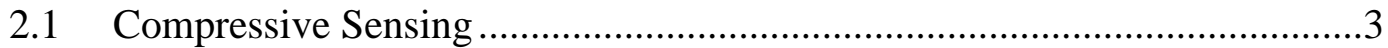

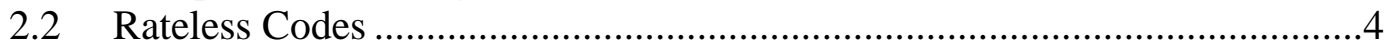

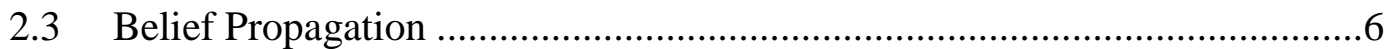

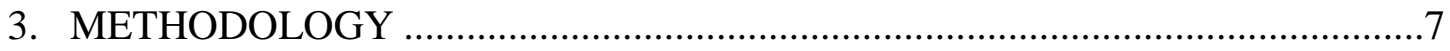

3.1 Compressive Sensing based Image Compression ..........................................

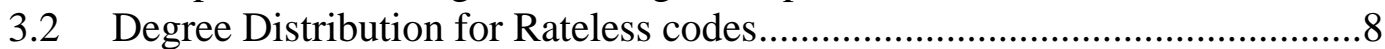

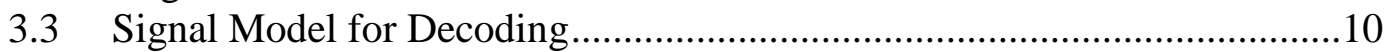

3.4 Image Reconstruction via Belief Propagation ...............................................12

4. SIMULATION RESULTS ………………………......................................16

5. DETECTION OF HIDDEN IMAGES USING COMPRESSIVE SENSING.......19

5.1 Compressibility test to detect hidden images in Covert Channel ................19

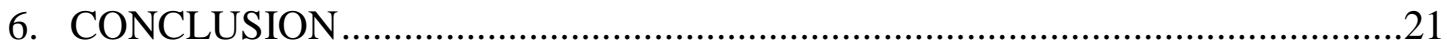

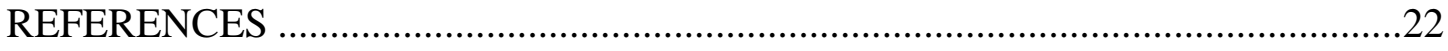

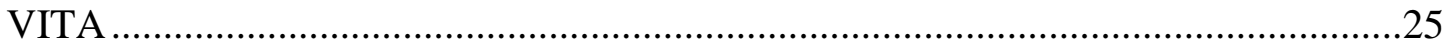




\section{LIST OF FIGURES}

1 Image encoding using CS ................................................................

2 Generating measurements ' $Y$ ' using ' $G$ ' and sparse vector ' $a$ '

3 Parallel channel scheme used in CS-based encoding …….............................10

$4 \quad$ Gaussian mixture model for signal coefficients..............................................12

$5 \quad$ Bipartite graph denoting the image pixel nodes ' $X$ ' .....................................13 and measurement node ' $Y$ '

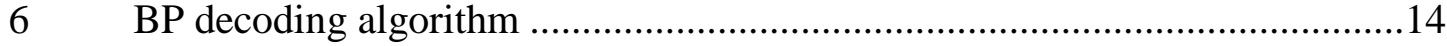

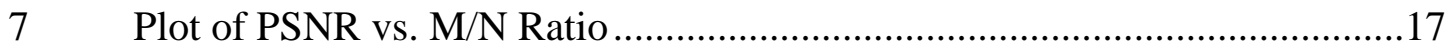




\section{LIST OF ABBREVIATIONS}

MDC - Multiple Description Coding

CS - Compressive Sensing

BP - Belief Propagation

DCT - Discrete Cosine Transform

DWT - Discrete Wavelet Transform

SWT - Stationary Wavelet Transform

LT - Luby Transform

LDPC - Low Density Parity Check

IID - Independent and Identically Distributed

SRP - Sparse Random Projection

DRP - Dense Random Projection

PSNR - Peak Signal to Noise Ratio

MSE - Mean Square error

$\mathrm{dB}$ - Decibel

pmf-Probability Mass Function

$p d f-$ Probability Density Function 


\section{LIST OF SYMBOLS}

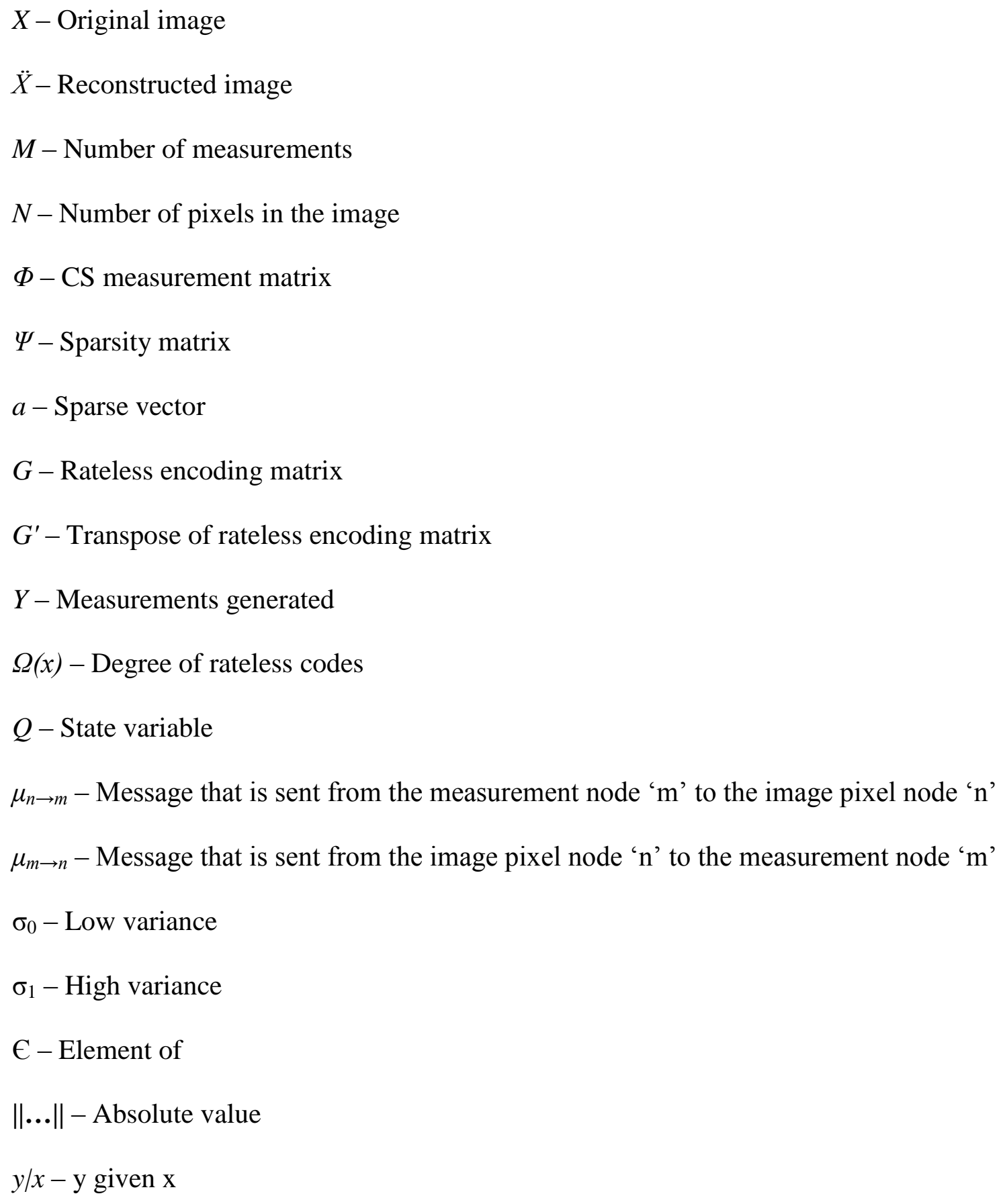


$S$ - Sparsity rate

$q$ - Channel size

$\delta$ - Error probability 


\section{CHAPTER 1 INTRODUCTION}

Image processing has an increasing demand due to its diverse application in areas such as multimedia computing, biomedical imaging, secured image data communication, content based image retrieval, etc. It is a challenging task to impart such applications to a machine in order to integrate and interpret the visual information embedded in still images, graphics and video or moving images in our visually fascinating world. Therefore, it is very essential to understand the techniques of storage, processing, transmission, recognition and interpretation of images. One of the simplest and best methods with which we can store and transmit the images is image compression. The advantage of image compression is that it reduces the visual redundancies in data while protecting the critical features intact in order to represent the image frames with significantly lesser number of bits and hence condenses the requirements for storage and effective communication bandwidth.

Generally, image compression algorithms transform high resolution images into images of lower resolution, while keeping the crucial features intact. Thus, a large digital set of data is converted into a relatively smaller one. An image compression technique would prove remarkable if there is a way to incorporate the data compression directly by avoiding the large set of digital data to begin with. This can be achieved through CS.

In recent times, CS has been the subject of interest due to its ability to recover a signal or in our case, an image from a very few samples utilizing the prior knowledge that the image is sparse (i.e. 
having a very few non-zero coefficients) or compressible in nature. Thus the storage and the encoding complexity are greatly reduced as the image is sampled in a reduced dimensional space. CS determines the weighted linear combination of samples i.e. measurements (also known as multiple descriptions or projections) in a basis in which the image is said to be sparse. In order to generate the measurements, a random matrix is the best choice [1]. Rateless codes are a class of codes that can generate potentially limitless number of output symbols from a fixed number of message symbols. Rateless codes are the most efficient choice for a random matrix as it does not consider the rate of the channel. Thus, we employ non uniform rateless codes along with the sparse image vector in order to generate the measurements. Measurements that are generated can be decoded using the BP decoding algorithm in order to reconstruct the image. We employed BP decoding algorithm which uses iterative message passing technique [2], [3] and [4] over other decoding algorithms like $l_{0}$ norm optimization, $l_{l}$ norm optimization [5], greedy decoding [13] etc. as these algorithms have certain drawbacks like solving $l_{0}$ norm optimization is NP hard [4], $l_{1}$ norm optimization method is generally known to have cubic computational complexity [4] and encoding by a dense Gaussian $\Phi$ is slow [2]. 


\section{CHAPTER 2}

\section{LITERATURE REVIEW}

\subsection{Compressive Sensing}

Compressive sensing, also termed as compressed sensing, compressive sampling or sparse recovery, is an efficient technique in the field of sampling theory. The foremost advantage of CS is that it requires far fewer samples than that required by the Nyquist rate in order to reconstruct the image i.e. CS theory has the ability to reconstruct an image of length $N$ that is known to be sparse with $M$ measurements where $M<<N$. Thus, we can reduce the storage and computational complexity. Also, the CS technique is less complex in the encoding and decoding processes and its ability to correlate to data is remarkable. Compression of the image is obtained by merely storing only the largest basis coefficients which convey more information compared to coefficients with lesser values as the reconstruction of the image mainly depends on those high valued coefficients.

The main idea behind CS is to determine the weighted linear combination of pixels, also called the measurements in a basis different from the one in which the image is sparse. By exploiting the sparsity of the image, the number of measurements required for full recovery can be reduced significantly. Mathematically, CS is a signal in a sparse basis induced by vectors $\left\{\Psi_{i}\right\}_{i=1}^{N}$ or the sparsity matrix $\Psi$ (also termed as transform basis) and can be represented as $X=\sum_{i=1}^{N} \Psi_{i} a_{i}$ or $X=\Psi a$ is called $K$-sparse if only $K$ values of $a_{i}$ are non-zero and $K<<N$. 
An image $X \in R^{N}$ has a sparse representation in one basis i.e. $X=\Psi a$, where $\Psi$ is the sparsity matrix (i.e. transform basis) and $a$ is the sparse vector. In this work, we employ the Discrete Cosine Transform (DCT) [5] to be the transform basis but in general the transform basis can be any one of the transform techniques like Discrete Wavelet Transform (DWT), Stationary Wavelet Transform (SWT) etc. This image can be recovered from a small number of measurements onto a second basis that is incoherent with the first one. In the real world, an image by itself is not sparse, meaning that the majority of the coefficients which represents the image will be non-zero. The image can be made sparse or compressible by using any one of the transform techniques mentioned above. An alternative approach to obtain sparse image is by using DCT, DWT or SWT sparsity matrices instead of the transform itself.

According to [1], for an image $X$ of length $N$ which is $K$ sparse and $K<<N$, CS theory states that $M=O(K \log (N / K))$ random linear projections or measurements of $X$ are sufficient to robustly reconstruct the image at the decoding end.

\subsection{Rateless Codes}

Rateless codes, also known as Luby transform (LT) codes [6], Raptor codes [7], or online codes [8], are a class of codes that can generate potentially limitless number of output symbols from a fixed number of message symbols using the generator matrix, or the rateless encoding matrix, $G$. Additionally, more encoding symbols can be generated as and when the need arises and transmitted over the channel until the decoder has adequate number of symbols in order to recover the data. On contrary to the traditional codes, rateless codes on lossy channels do not assume any knowledge about the channel. Therefore, rateless codes prove to be very appropriate in the applications when the channel erasure probability is unknown [16]. 
Most of the entries in the rateless encoding matrix $(G)$ are dominated by zeros while the non-zero entries are sparse independent and identically distributed (IID) Gaussian or Bernoulli (+1) vectors. These entries provide a useful universal measurement basis for CS [10]-[12]. As long as the rateless encoding matrix $G$ is not too sparse, the measurements will succeed in capturing sufficient information about $X$ to decode the image.

The output symbols are generated independently and arbitrarily such that each output symbol will be the sum of certain input symbols. From the summation of the input symbols, the degree distribution and also the neighboring nodes can be determined. It is essential that the decoder knows the degree distribution and the set of neighbors of each encoding symbol when the encoding symbols are used to recover the original image. Asymptotically, a good degree distribution for the rateless codes was developed and is introduced in [6], [7] and [8]. The encoding and decoding processes are faster in case of rateless codes, because multiplying a signal by a sparse generator matrix is fast. The design of rateless encoding matrix $G$, characteristics such as column and row weights i.e. degree distribution rely upon the relevant signal, measurement model and the decoding algorithm that is employed. The degree distribution plays a crucial role in determining the success of the decoder [7]. The performance of non-uniform rateless codes, meaning rateless codes having irregular degree distribution is known to be superior compared to uniform rateless codes [9]. Therefore, in our work, we have employed non-uniform rateless codes.

The mathematics behind the degree distribution of rateless codes is $\Omega(x)=\sum_{i=1}^{N} w_{i} x^{i}$, where $w_{i}$ the probability is that degree $i$ is chosen and $x$ represents the entries in the rateless encoding matrix $G$. The encoding process involves generating independent and random measurements from the degree distribution $\Omega(x)$ to obtain a weight between 1 and $N$. After generating independent and random descriptions, a vector $V=\left(v_{1}, v_{2} \ldots v_{n}\right)$, with a weight of $w$ is 
chosen at random. Finally, the value of the encoded symbol is calculated by the formula $\sum_{i=1}^{N} v_{i} X_{i}[18]$

\subsection{Belief Propagation}

$\mathrm{BP}$ is a decoding technique that is used to reconstruct the image from the set of generated measurements. BP algorithm involves an iterative message passing technique in which neighboring variables talk to each other passing messages. BP algorithm basically solves inference problems based on an iterative message passing technique [2]-[3]. After enough iterations, this series of conversations is likely to converge to a consensus that determines the marginal probability of all the variables. Estimated marginal probabilities are called "Beliefs". BP algorithm updates messages until convergence and then calculates beliefs. Once we have the measurements at the decoding end, the BP decoding algorithm is applied to a bipartite graph (which is discussed in detail in Chapter 3) with the image pixel nodes on one side and the measurements on the other side. CS-BP decoding algorithm uses $O\left(N \log ^{2}(N)\right)$ computations [2]. The application of CS for BP is discussed in [2] and [3]. 


\section{CHAPTER 3}

\section{METHODOLOGY}

\subsection{Compressive Sensing based Image Compression}

In this algorithm (i.e. compressive sensing based image compression) the image is compressed and represented as a sparse signal in a reduced dimensional space. Once we have the sparse image, we can generate measurements using the CS measurement matrix $(\Phi)$. This constitutes the encoding aspect of the process.

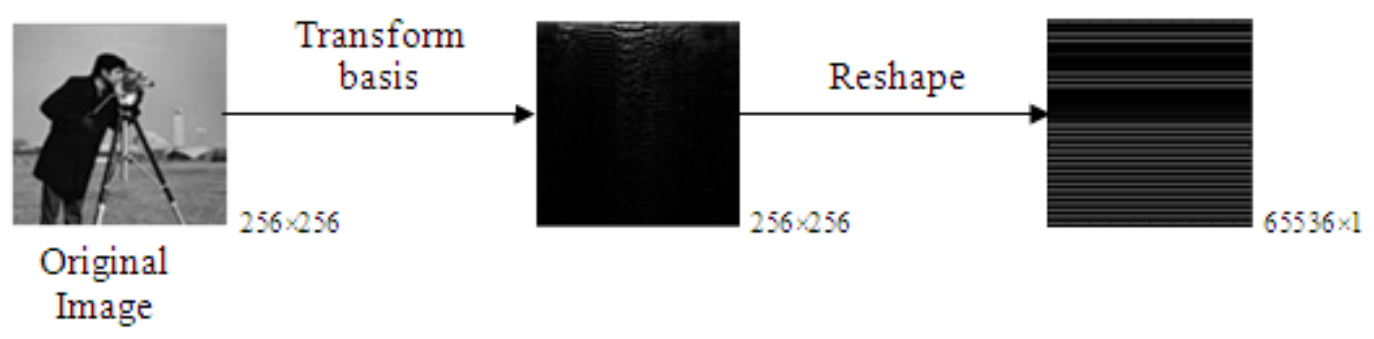

Fig 1: Image encoding using CS.

The CS measurement matrix $(\Phi)$ is obtained from the rateless encoding matrix $G$ and the transpose of the sparsity matrix $\Psi^{\prime}$. It is necessary that the rateless encoding matrix $G$ is strictly sparse in nature, whereas, the measurement matrix $(\Phi)$ may be obtained by either of the CS algorithms, i.e. sparse random projection (SRP) or dense random projection (DRP).

As sparsity is a crucial factor in CS based recovery, we choose SRP in our work. Encoding complexity is low in case of a SRP when compared to DRP. Also the incoherency between rateless encoding matrix $G$ and the transform basis $\Psi$ must be minimized [17] meaning 
that both $G$ and $\Psi$ must be generated randomly so that the image pixels values can be recovered error free at the decoding end. CS theory requires $M=O(K \log (N / K))$ measurements in order to reconstruct the original image with high probability.

In order to encode the image, measurements $Y$ at the encoding end are generated using the CS measurement matrix $\Phi$ and the sparse image signal $X$ as follows,

a. When the transforms technique is employed:

$Y_{m x l}=\Phi_{m x n} \times X_{n x l}=\Phi \times \Psi a$; where $\Phi$ is the CS measurement matrix generated from $G$.

b. When the sparsity matrix is employed:

$Y_{m x l}=\Phi_{m x n} \times X_{n x l} ;$ where $\Phi=G \Psi^{\prime}$

$Y_{m x l}=G \Psi^{\prime} \Psi a=G a$; where $G$ is the rateless encoding matrix and $a$ is sparse vector.

$$
\text { Measurements }(\mathrm{Y})_{\mathrm{m} \times 1}=\left[\begin{array}{ccc}
G_{11} & \cdots & G_{1 n} \\
\vdots & \ddots & \vdots \\
G_{m 1} & \cdots & G_{m n}
\end{array}\right]_{m \times n} \times
$$

Fig 2: Generating measurements ' $Y$ ' using ' $G$ ' and sparse vector ' $a$ '.

When $\Psi$ and $\Psi^{\prime}$ are orthonormal, $\Psi^{\prime} \Psi=I$ and thus the measurement matrix will contain $G a$. These measurements generated serve as the input to the BP decoder which aims at reconstructing the image.

\subsection{Degree Distribution of Rateless Codes}

The crucial step in the encoding and measurement generation process is when the image node randomly selects a weight from the degree distribution $\Omega(x)$. Depending on the design of the degree distribution $\Omega(x)$, we can determine whether the measurements are SRP or DRP. 
DRP allows more accurate recovery of a sparse signal at the decoding end as compared to SRP but the computational complexity of DRP is very high. Thus, it is our goal to intelligently design the degree distribution $\Omega(x)$ such that only SRP are generated. In order to design the degree distribution $\Omega(x)$ such that it generates only sparse projections, we use a parallel channel scheme. The application of channel coding for CS has been studied earlier in [2], [14] and [15].

Next, it is essential to determine how to design the degree of the rateless code such that the implementation of CS is practical and the performance of CS is optimum. The authors in [2] have employed a fixed degree for all measurements and from the simulation results it can be seen that the optimum value of $\Omega(x)=w$. In our work, we propose employing a non-uniform degree for rateless codes as non-uniform rateless codes are known to perform better when compared to uniform rateless codes [9]. Thus, CS is modeled with a set of parallel channels. This model is designed in [19] and its application has been studied for imaging.

The main aim in CS is to reconstruct all the values of the image pixels after receiving $M$ measurements. The received measurements can be considered as parity bits as those bits help the decoding end to reconstruct the original $K$-sparse vector. This $K$-sparse vector can be modeled as input to a ' $q$-ary' symmetric channel $(q-S C)$ whose output is a vector comprised of zero's, i.e. an all zero vector. A ' $q$-SC' channel with a size of $q$ and having an error probability $\delta$ can be defined as follows,

$$
\operatorname{Pr}(y \mid x)= \begin{cases}1-\delta & \text { if } y=x \\ \delta /(q-1) & \text { otherwise }\end{cases}
$$

where $x, y \in \mathrm{F}_{\mathrm{q}}$ are the input and output of the channel respectively [15]. The ' $q$-ary' symmetric channel modeled in this work has an error of $\frac{K}{N}$. As the parity bits or the measurements received 
are error free, it implies that the parity bits can be modeled as an ideal channel scheme whose output is the same as input.

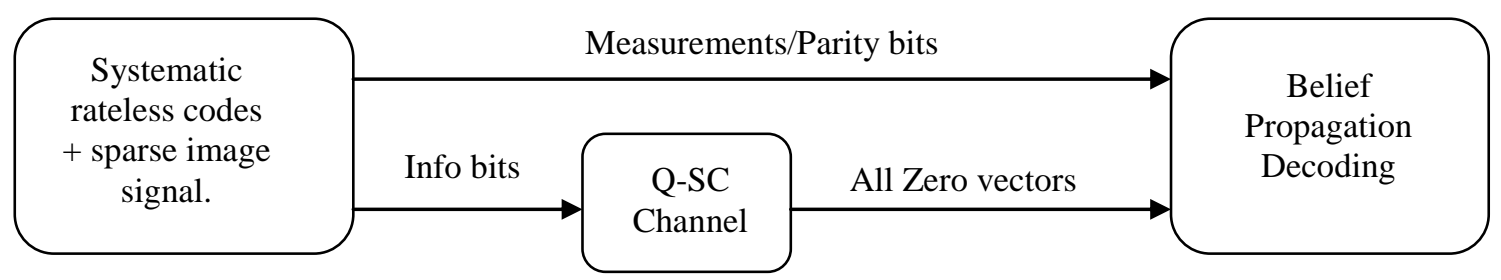

Fig 3: Parallel channel scheme used in CS-based encoding.

Fig 3 shows the parallel channel model used in CS based encoding. For large values of $q$, the capacity of this channel can be calculated by $C_{q-S C}=1-\delta$. This set of parallel channels is used in order to design the degree distribution. In order to design the rateless codes for the channels as shown in Fig 3, we consider both the $q$-SC channel and the parity bits and base the code design on a set of non-uniform channels where different bits encounters varying noise levels. Thus, CS uses two different degree distribution $\Omega_{1}(x)$ and $\Omega_{2}(x)$. Based on this degree distribution, a random realization of $G$ can be generated. By using Gaussian elimination, we are then able to construct the generator matrix $G^{\prime}$ that is used for rateless coding. Lastly, the measurement matrix $\Phi$ is obtained by removing the submatrix identity from $G^{\prime}$.

\subsection{Signal Model for Decoding}

As a mixture of Gaussian models has proven to be efficient in modeling real world signals, they can be effectively employed in image processing [2]. Thus, we have considered a two-state Gaussian mixture model as a prior that interprets the prior knowledge regarding the sparsity of the image signal. From the previous section, we know that $X=[X(1), X(2), \ldots . ., X(N)]$ $\in R^{N}$, and consider the signal $x=[x(1), x(2), \ldots \ldots x(N)]$ to be the result of $X$. In order to model this 
signal, we need two probability functions i.e. Probability Mass Function (pmf) and Probability Distribution Function ( $p d f)$ [2]. The Probability Distribution Function ( $p d f)$ of state variable $\mathrm{Q}=1$ or $\mathrm{Q}=0$ represents the probability that $X$ has either a large coefficient or a small coefficient and the Probability Mass Function (pmf) models the small and the large coefficients with zero mean Gaussian mixture model with high and low variances

Let $Q=[Q(1), Q(2) \ldots Q(N)]$ denote the state random vector associated with the image signal. In order to make sure that we have $K$ large coefficients approximately, we consider $p d f$ of the state variable $Q(i)$ to be Bernoulli $(+1)$ which can be represented as below,

$$
\operatorname{Pr}(Q(i)=1)=S \text { and } \operatorname{Pr}(Q(i)=0)=1-S
$$

where $S=\frac{K}{N}$ is the sparsity rate.

As the estimated sparse image signal contains majority of small coefficients (zero or close to zero) and a very few large coefficients which carry some information, we can relate the $p d f$ of each coefficient $f(X(i))$ along with a state variable $Q(i)$ that can take two values. These coefficients can be represented by zero mean Gaussian distributions with high variance denoted by $Q(i)=1$ and low variance denoted by $Q(i)=0$ as follows.

$$
\left.f(X(i)) \mid Q(i)=1) \sim N\left(0, \sigma_{1}^{2}\right) \text { and } f(X(i)) \mid Q(i)=0\right) \sim N\left(0, \sigma_{0}^{2}\right)
$$

where $\sigma_{1}{ }^{2}>\sigma_{0}{ }^{2}$. In the presence of the side information, this prior model can be altered based on the information available.

Fig 4 shows the distribution of the original image signal $X$ based on the state variables $Q$ $=0$ and $Q=1$. The state Gaussian mixture model is depends on parameters such as sparsity rate $S$, the variances $\sigma_{1}^{2}$ and $\sigma_{0}^{2}$ of the Gaussian $p d f$ 's which corresponds to the states. 


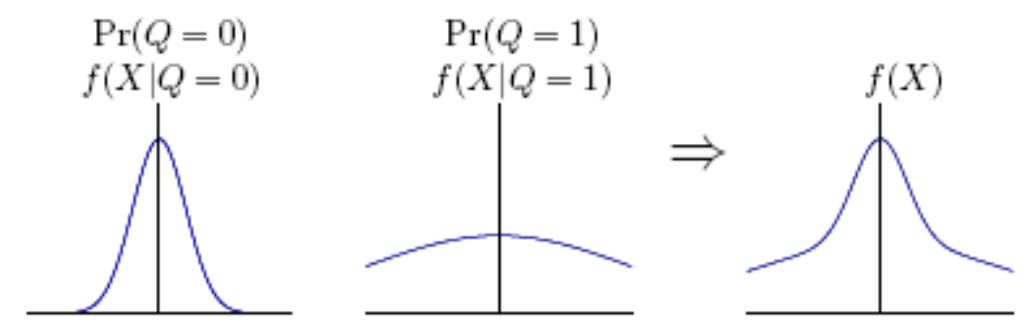

Fig 4: Gaussian mixture model for signal coefficients.

As mentioned earlier, in our work, we have assumed that all the unknown variables have the same prior distribution as we do not have any side information available at the decoding end during the initiation of the decoding process.

\subsection{Image Reconstruction via Belief Propagation}

$\mathrm{BP}$ is a decoding algorithm which involves iterative message passing technique in which the neighboring nodes talk to each other passing messages. After enough iteration, this series of conversations is likely to converge to a consensus that determines the marginal probability of all the variables. Estimated probabilities are termed as "Beliefs". BP algorithm updates messages until convergence and then calculates the beliefs. Once we have the measurements at the decoding end, BP decoding algorithm is applied to a bipartite graph with the image pixel nodes on one side and the measurements on the other side.

Fig 5 clearly explains how measurements $Y$ can be generated from the image pixels $X$ and the CS measurement matrix $\Phi\left(\Phi=G \Psi^{\prime}\right)$. Once the decoder has the measurements, BP algorithm uses the iterative message passing technique in order to solve the bipartite graph and reconstruct the image. As mentioned in chapter 2, CS-BP algorithm uses $O\left(N \log ^{2}(N)\right)$ computations. The main advantage of CS-BP algorithm is that the prior information can be easily incorporated which aids in the decoding process. In CS-BP algorithm, a two state mixture Gaussian model is used as 
the prior information for all variable nodes, where all the nodes are considered to be zero-mean Gaussian random variables.

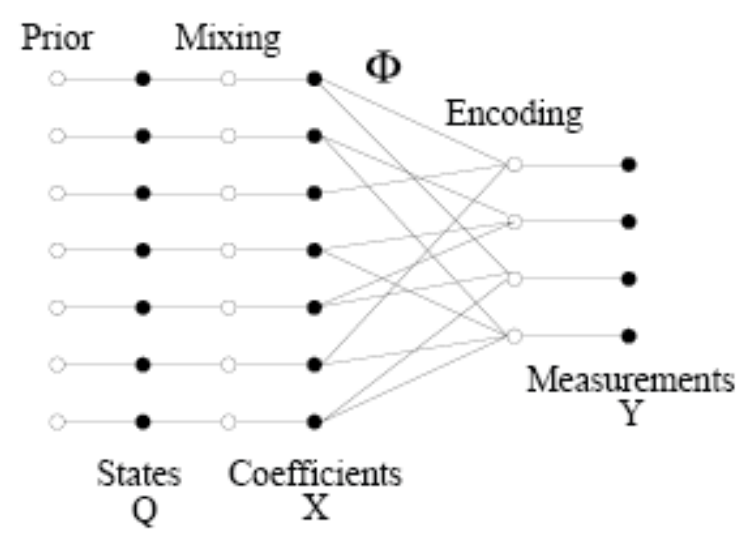

Fig 5: Bipartite graph denoting the image pixel nodes ' $X$ ' and measurements ' $Y$ '

The message that is sent from image pixel node $n$ to one of its neighbors i.e. the measurement node $\mathrm{m}$ is denoted by $\mu_{n \rightarrow m}$ and the message that is sent from the measurement node $\mathrm{m}$ to the image pixel node $\mathrm{n}$ is denoted by $\mu_{m \rightarrow n \text {. }} \mathrm{BP}$ algorithm approximates the marginal distribution of the coefficient and state variables in the bipartite graph, conditional on any measurement $Y$. This is by iteratively passing the messages from the image pixel node and the measurement node. The input for the message passing process begins with input consisting of the prior probability function (pdf-prior) of the image pixel values. This value of pdf-prior is sent from the image pixel node to the measurement node on the bipartite graph. The updated messages are sent back to the image pixel node by using the constraint on the set of the image pixel nodes generated by the measurement nodes. Iterative BP decoding algorithm repeats the iterations until it satisfies the maximum number of iterations (maxiter) and then the messages calculate the beliefs. The main difference between BP decoding algorithm in our work and the BP decoding algorithm that is proposed in [2] is that in our work, the measurement matrix is 
composed of 0's and 1's whereas in [2], the measurement matrix is composed of 0's and \pm 1 's and in our work, the degree distribution in the measurement matrix is non-uniform whereas in [2], the degree distribution is uniform i.e. they have fixed column and row degrees

The BP algorithm is summarized as shown in fig 6. In the algorithm, neigh (n) denotes the image pixel node and the neigh (m) denotes the measurement pixel node, con (neigh $(\mathrm{m})$ ) is the constraint on the set of image pixel nodes neigh (n).

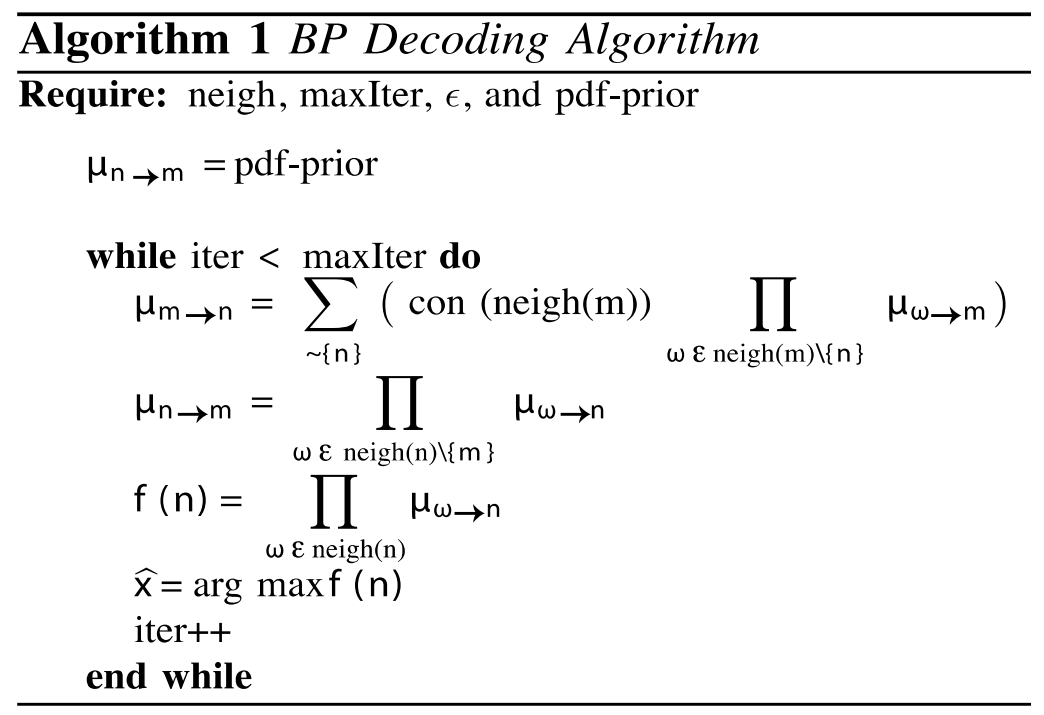

Fig 6: BP decoding algorithm.

In the above BP algorithm, the very first step is to initialize the $p d f$-prior. The $p d f$-prior initializes the message sent from the image pixel node to the measurement node. We know that, for a sparse image, a large number of coefficients are zero or close to zero which is negligible and a small number of its coefficients are large valued. In order to model this behavior accurately, we must consider the two probability functions discussed in section 3.3 i.e. $p d f$ and pmf. 
In order to make sure that we have $K$-sparse signal, $p d f$ must satisfy the condition that $\operatorname{Pr}(\mathrm{Q}(\mathrm{i})=1)=S$ and the $\operatorname{Pr}(\mathrm{Q}(\mathrm{i})=0)=1-S$ and pmf must satisfy the condition $f(X(i) \mid Q(i)=$ 1) $\sim N\left(0, \sigma_{1}{ }^{2}\right)$ and $f(X(i) \mid Q(i)=0) \sim N\left(0, \sigma_{0}{ }^{2}\right)$ where $\sigma_{1}>\sigma_{0}$. This is the two state mixture Gaussian model and the pdf-prior can be designed as follows which denotes that the probability that each node is significant is $(K / N=S)$, which represents the sparsity of the vector and the variances of all the significant nodes is $\sigma_{1}{ }^{2,}$ and the variances of all the insignificant nodes is $\sigma_{0}{ }^{2}$. Thus the pdf-prior can be denoted as

$$
\text { pdf-prior }=(K / N) N\left(0, \sigma_{1}^{2}\right)+(1-K / N) N\left(0, \sigma_{0}^{2}\right)=(S) N\left(0, \sigma_{1}^{2}\right)+(1-S) N\left(0, \sigma_{0}^{2}\right)
$$

In case of the presence of side information, this pdf-prior can be altered based on the information available. 


\section{CHAPTER 4}

\section{SIMULATION RESULTS}

In order to demonstrate the efficiency of the proposed compression and decoding algorithm, we applied the algorithm on the 'cameraman' image. The size of the original image frame is $256 \times 256$. In order to make the computation faster, the original image frame was divided into 16 sub images of frames $64 \times 64$. This work has been carried out under the assumption that there is no transmission error. Our simulations were carried out in MATLAB.

As discussed in the previous chapter, the performance of the non-uniform rateless codes is superior compared to uniform rateless codes, therefore the degree distribution used in our code in order to generate the encoding matrix is as follows:

$$
\Omega(x)=0.25 x^{5}+0.25 x^{10}+0.5 x^{15}
$$

Parameters of the two state Gaussian mixture model $\sigma_{0}{ }^{2}$ and $\sigma_{1}{ }^{2}$ are chosen to be 1 and 10 respectively. Since each sub frame is of dimension $64 \times 64$, the total number of pixels in each iteration is $N=64 \times 64=4096$ and the total number of pixels in entire image is 65536 , the

maximum iterations i.e. maxIter $=10$. The sparsity of the image i.e. $S=\frac{K}{N}$ is assumed to be 0.1 initially.

Processing an image may cause loss of information and might degrade the quality of the reconstructed image. In order to evaluate the performance of the reconstructed image with respect to the original image, we compute Peak Signal to Noise Ratio (PSNR) [4], [5] and [20]. 
PSNR is an image quality metric which determines the information loss or degrade in quality by objective method i.e. by comparing explicit numerical criteria [21] and [22].

The PSNR is given by:

$$
P S N R=20 \log _{10}(255 \times 256) /\left\|(X-\ddot{X})^{2}\right\| \text { in } \mathrm{dB}
$$

Where $X$ is the original signal, $\ddot{X}$ is the reconstructed signal and $\left\|(X-\ddot{X})^{2}\right\|$ is the mean square error (MSE). For a two monochrome images $I$ and $P$ of dimension $x \times y$ MSE is given by,

$$
M S E=\frac{1}{x \times y} \sum_{i=0}^{x-1} \sum_{j=0}^{y-1}[I(i, j)-P(i, j)]^{2}
$$

It can be clearly seen that the values of the PSNR approaches infinity as the MSE approaches zero which implies that lower the error, higher will be the value of the PSNR which provides a better quality image. On the other hand, if the value of the PSNR is very low, it implies that the numerical difference between the reconstructed image and the original image is very high and thus the image quality of the reconstructed signal is low.

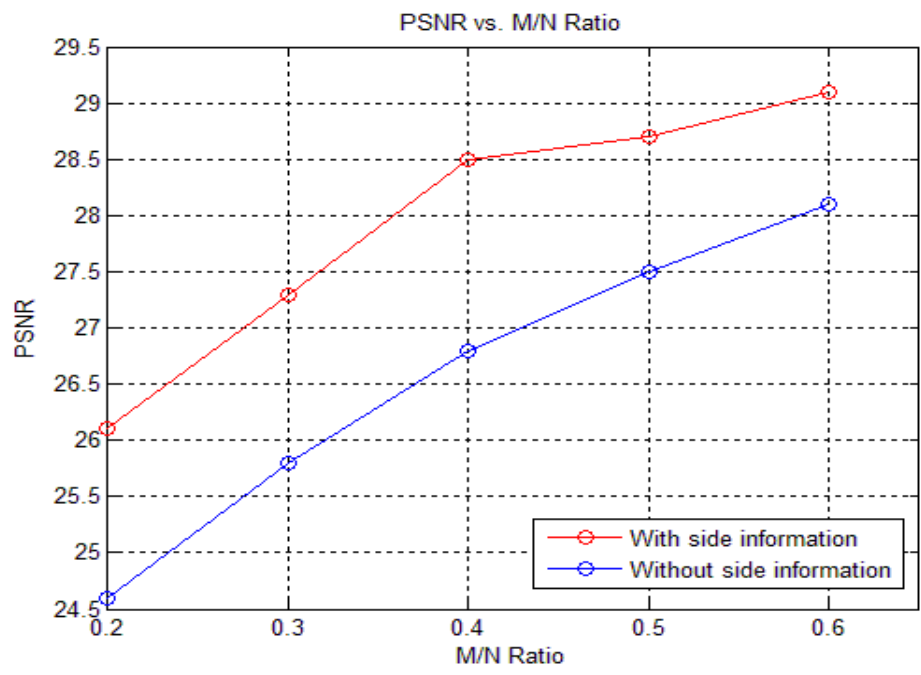

Fig 7: Plot of PSNR vs. M/N Ratio 
Fig 7 illustrates the plot for PSNR vs. Number of measurements for the 'cameraman' image of frame size $256 \times 256$. We calculate the PSNR for each sub frame of size $64 \times 64$ and compute the overall PSNR by averaging the PSNR values of all sub frames. Considering the entire image, we start with $20 \%$ i.e. 13107 measurements and go up to $60 \%$ i.e. 39321 measurements and the corresponding PSNR values are determined. Thus it is evident that as the number of measurements increases, error decreases. 


\section{CHAPTER 5}

\section{DETECTION OF HIDDEN IMAGES USING COMPRESSIVE SENSING}

\subsection{Compressibility test to detect hidden images in Covert Channel}

In the previous chapters, we have studied how CS can be applied in image processing. In this chapter, we study another application of CS i.e. its ability to detect a hidden or a covert channel [23]. A covert channel is a means of transmitting information in various ways such that it is difficult to detect that the transmission is taking place. Some of the techniques of detecting a covert channel are based on the delay experienced in the inter packet arrival times, based on the change in the header or payload information in the packets etc. [23] and [24]

In the previous chapters, we studied how the images are compressible. In general a signal will be compressible in nature if the coefficients which represent the signal are correlated. Thus when the image can be compressed, it implies that the pixels which represent the image will be correlated and when compressed gives us a prior knowledge of the image structure. This prior knowledge can helps in the detection of the hidden image. The presence of a hidden image will alter this structure of the original image because of the additional information it carries. This detection technique mainly depends on the fact that a set of values with a high degree of regularity or correlation is more compressible than a set of values with a high degree of randomness. Thus, by compressing the image in the presence of a hidden image gives us different compression values when compared to the compression values of the pixels in the original image. 
The error between the original image and the reconstructed image is calculated by Peak Signal to Noise Ratio (PSNR) which is discussed in the previous chapter. When the original image is reconstructed in the absence of the hidden image, we measure the PSNR to determine its performance. When the original image is reconstructed in the presence of the hidden image, it is noted that the PSNR value decreases when compared to the PSNR value obtained in the absence of the hidden image. Higher the value of the PSNR better is the reconstruction quality and vice versa. Therefore, the results clearly imply that there is degradation in the quality of the image and that the original image has been tampered. Thus, the presence of hidden image can be determined. 


\section{CHAPTER 6}

\section{CONCLUSION}

In this work, we proposed an algorithm for CS-based BP which is a compression and decoding algorithm for image processing. This CS encoding algorithm successfully compresses the signal at a rate less than that required by the Nyquist rate and the proposed $\mathrm{BP}$ decoding algorithm perfectly reconstructs the signal from a very small subset of samples which carry the necessary information. Thus, the main advantage of employing BP over other decoding algorithms like $l_{0}$ norm optimization, $l_{l}$ norm optimization, greedy decoding, etc., is that the BP algorithm is extremely general and can be applied to any graphical model. The BP algorithm provides an exact solution when there are no loops in the graph, such as a tree, and in the presence of loops BP provides approximate solution. Lastly, decoding is faster compared to the other the decoding algorithms mentioned above.

Our results show that the approach is promising because in the proposed algorithm, we aim at reconstructing the image without having any side information. However, it can be noted that the results improve in the presence of the side information and the reconstruction quality can be improved. In majority of the cases, it is not possible to have the side information about the image. Therefore, the proposed CS-BP algorithm proves to be a general case to reconstruct the image in the absence of any side information. Also, the proposed CS-BP algorithm can be extended and generalized to video coding as well. 


\section{REFERENCES}

[1] D.L. Donoho, “ Compressed Sensing," IEEE Trans. Information theory, vol. 52, no. 4 pp. 1289-1306, April 2006.

[2] D. Baron, S. Sarvotham, R.G. Baraniuk, "Bayesian Compressive Sampling via Belief Propagation”, IEEE trans. Signal processing, volume 58, no. 1, pp.269-280, 2010.

[3] W. Xu and B. Hassibi, "Further results on performance analysis for compressive sensing using expander graphs," In proc of the Forty-First Asilomar Conference on Signals, Systems and Computers, pp. 621-625, 2007.

[4] Beigi, P, Xiaoyu Xiu, Jie Liang, "Compressive sensing based multiview image coding with belief propagation," In proc of the $43^{\text {rd }}$ Asilomar Conference on Signals, Systems and Computers, pp. 430-433, 2010.

[5] Justin Romberg, "Imaging via Compressive sampling," IEEE Signal Processing Magazine, vol.25, pp. 14-20, March 2008.

[6] M. G. Luby, "LT codes," In proc. of the $43^{\text {rd }}$ IEEE symposium on the Foundations of Computer science (STOC), pp. 271-280, 2002.

[7] A. Shokrollahi, "Raptor codes," In proc. of the IEEE symposium on Information Theory, p.36.2004.

[8] P. Maymounkov, “Online codes,” NYU Technical Report TR2003-883, 2002.

[9] M. G. Luby, M. Mitzenmacher, M. A. Shokrollahi, and D. A. Spielman, "Efficient erasure correcting codes," IEEE Transactions on Information Theory, vol. 47, no. 2, pp. 569-584, February 2001.

[10] E. Candes and T. Tao, "Robust uncertainity principles: Exact signal reconstruction from highly incomplete frequency information," IEEE transactions on Information Theory, vol. 52, no. 2, pp. 489-509, 2006. 
[11] S. Chen, D. Donoho, and M. Saunders, "Atomic decomposition by basis pursuit,", SIAM Journal on Scientific Computing, pp. 33-61, 1998.

[12] W. Wang, M. Garofalakis, and K. Ramachandran, "Distributed sparse random projections for refinable approximation," In proc of the ACM/IEEE International Symposium on Information Processing in Sensor Networks, pp. 331-339, 2007.

[13] M. F. Duarte, M. B. Wakin, and R. G. Baraniuk, "Fast reconstruction of piecewise smooth signals from random projections," In Proc. of Signal Processing with Adaptive Sparse Structured Representations, 2006.

[14] M. S. Asif, W. Mantzel, and J. Romberg, "Random channel coding and blind deconvolution," In Proc. Allerton conference on communication, control and computing, 2009.

[15] F. Zhang and H. D. Pfister, "Compressed sensing and linear codes over real numbers," In Proc of information theory and applications, Feb 2008.

[16] Mina Sartipi, "Modern Error Control Codes and Application to Distributed Source Coding," PhD Dissertation, Georgia Institute of Technology.

[17] P. Ramachandra, M. Sartipi, "Compressive Sensing based Imaging via Belief Propagation," In Proc of 45 ${ }^{\text {th }}$ Asilomar Conference on Signals, Systems and Computers, 2011.

[18] Mina Sartipi, Rob Fletcher, "Energy-Efficient Data Acquisition in Wireless Sensor Networks using Compressed Sampling," In proc of Data Compression Conference, March 2011.

[19] Mina Sartipi, "Compressive Sensing with Encoder Side Information," Submitted to IEEE Globecom, February 2011.

[20] A. Horé and D. Ziou, "Image Quality Metrics: PSNR vs. SSIM", In Proc. ICPR, 2010, pp.2366-2369.

[21] M .Cadik and P. Slavik, "Evaluation of two principal approaches to objective image quality assessment," $8^{\text {th }}$ International Conference on Information Visualization, IEEE Computer Society Press, pp. 513-551, 2004.

[22] T. B. Nguyen and D. Ziou, "Contextual and non-contextual performance evaluation of edge detectors," Pattern recognition letters, vol. 21, no. 9, pp. 805-81. 
[23] G. Garateguy, G. R. Arce, J. Pelaez, "Covert Channel Detection in VoIP streams," In proc of $45^{\text {th }}$ Annual conference on Information Sciences and Systems, 2011.

[24] V. Berk, A. Giani, and G. Cybenko, "Detection of Covert Channel Encoding Network Packet Delays," Tech. Rep. TR2005-536, Dartmouth College, Computer Science, Hanover, NH, August 2005. 


\section{VITA}

Preethi was born in Manchester, New Hampshire, USA and later moved back to India. She did her entire schooling in India. She attended Visveswaraiah Technological University, India and received her Bachelor of Engineering degree in Telecommunication. She moved back to the United States in 2010 to pursue higher education. She received her Master of Science in Electrical Engineering from the University of Tennessee at Chattanooga. She was employed as a Graduate research assistant at the University of Tennessee at Chattanooga and she was also working as an intern in the Nuclear Power Group at Tennessee Valley Authority. Preethi is an Indian classical dancer and she enjoys reading and volunteers in social service endeavors in her spare time. 